\title{
A rare case of ovarian vein thrombosis in a gestational trophoblastic neoplasia patient
}

\author{
In Young Kim, Seung Hyun Kim, In Taek Hwang, Joong Gyu Ha, Jae Ho Cha \\ Department of Obstetrics and Gynecology, Eulji University Hospital, Eulji University School of Medicine, Daejeon, Korea
}

Ovarian vein thrombosis (OVT) is a rare disease with complications that can be life-threatening. An ovarian vein thrombus in a gestational trophoblastic neoplasia (GTN) is an extremely rare condition that has not been previously reported in the literature. We report the case of a 23-year-old woman who presented with symptoms of amenorrhea for 15 weeks and 6 days along with intermittent lower abdominal pain. She was diagnosed with a hydatidiform mole, and a metastatic workup was scheduled. Abdominal computed tomography showed a right ovarian vein thrombus. She received methotrexate chemotherapy combined with oral anticoagulants. Complete radiological remission was obtained. During the 12-month follow-up period, no disease progression or recurrence was noted. Early recognition and detection of the condition are of the utmost importance. The differential diagnosis of OVT must be considered when there is unexplained abdominal pain, fever, and leukocytosis during the diagnosis and treatment of GTN. A high level of suspicion is required for prompt diagnosis of OVT.

Keywords: Gestational trophoblastic neoplasia; Venous thrombosis; Anticoagulants

\section{Introduction}

Ovarian vein thrombosis (OVT) is a rare disease that usually presents in the first week postpartum, occurring in around $0.18 \%$ of puerperal women [1]. It is also known to be associated with other conditions such as malignancies, pelvic inflammatory disease, inflammatory bowel disease, sepsis, and recent abdominal or pelvic surgery [2-8]. No cases of OVT have been reported in gestational trophoblastic neoplasia (GTN) patients to date. In rare cases, OVT can lead to fatal complications. Therefore, early diagnosis and prompt treatment are of importance. Here we report a case of OVT in a patient with GTN.

\section{Case report}

A 23-year-old woman (gravida 0, para 0) presented to the Obstetrics and Gynecology Department with symptoms of amenorrhea for 15 weeks and 6 days and intermittent lower abdominal pain. She had no other signs and symptoms. She had no recent history of travel, surgery, trauma, or hospitalizations, and she was not taking any oral contraceptives. One month before admission, she tested positive for urine human chorionic gonadotropin ( $\mathrm{hCG}$ ); however, she did not receive any antenatal screening.

Physical examination findings were unremarkable. Most of the laboratory data were within normal limits. Serum $\beta-h C G$ level was elevated to 200,000 milli-International Unit/mL. Results of basic hemostasis tests, including prothrombin time and international normalized ratio were found to be normal.

A transvaginal ultrasound (US) scan revealed a bulky uterus with honeycomb pattern in the endometrial cavity. There was no evidence of an intrauterine gestational sac or an ec-

Received: 2018.05.08. Revised: 2018.07.19. Accepted: 2018.08.12. Corresponding author: Seung Hyun Kim

Department of Obstetrics and Gynecology, Eulji University Hospital, Eulji University School of Medicine, 95 Dunsanseo-ro, Seo-gu, Daejeon 34824, Korea

E-mail: medicine00@eulji.ac.kr

https://orcid.org/0000-0002-9861-3889

Articles published in Obstet Gynecol Sci are open-access, distributed under the terms of the Creative Commons Attribution Non-Commercial License (http://creativecommons. org/licenses/by-nc/3.0/) which permits unrestricted non-commercial use, distribution, and reproduction in any medium, provided the original work is properly cited.

Copyright (C) 2019 Korean Society of Obstetrics and Gynecology 


\section{Obstetrics \& Gynecology Science}

In Young Kim, et al. A rare case of ovarian vein thrombosis

topic pregnancy, and both the adnexa were unremarkable. Subsequently she underwent a dilatation and curettage, and the tissue obtained was sent for a biopsy. She was diagnosed to have a hydatidiform mole based on the histological examination 1 week later. Her $\beta$-hCG level decreased to 43,996.2 $\mathrm{mIU} / \mathrm{mL}$. However, she did not return for follow-up a week later, as an outpatient. Two months later, she visited the outpatient department with abdominal pain. Her $\beta$-hCG level

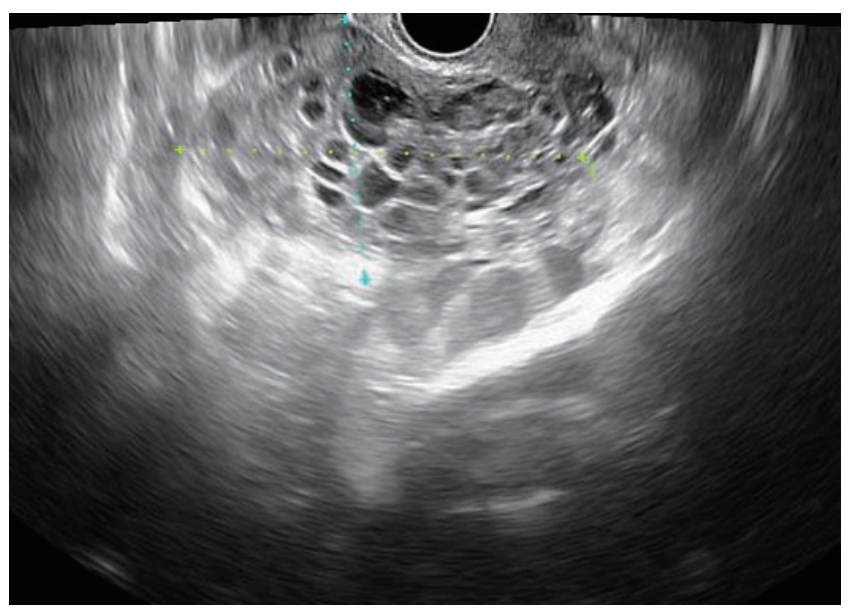

Fig. 1. Transvaginal ultrasound findings. The right adnexa showed multilobular and mixed echogenicity. again started increasing, reaching a level of 138,103.3 $\mathrm{mIU} / \mathrm{mL}$. A transvaginal US scan revealed no evidence of a bulky uterus and the endometrium showed a thickness of $0.5 \mathrm{~cm}$. However, a $9.1 \times 5.9 \mathrm{~cm}$ multilobular mass with mixed echogenicity was seen in the right adnexa (Fig. 1). A diagnosis of GTN was considered, and the required metastatic workup was performed. Abdominal computed tomography (CT) with intravenous contrast revealed a $10 \times 4.5 \mathrm{~cm}$ multilobular and tortuous tubular hypodense mass with strong enhancing septae and peripheral wall in the right adnexa (Fig. 2A). In addition, an $8.5 \times 8 \mathrm{~mm}$ right ovarian vein thrombus was revealed (Fig. 2B).

The patient was diagnosed as having stage II GTN and was assigned a low-risk score using the scoring system based on prognostic factors. She was treated initially with the 8-day methotrexate (MTX)-folinic acid (FA) regimen followed by multidose MTX. She also began oral anticoagulation therapy with rivaroxaban following consultation with the vascular surgery department. Following 5 courses of MTX-FA and 4 courses of 5 -day MTX therapy, the $\beta$-hCG values reached a normal level of $2.6 \mathrm{mlU} / \mathrm{mL}$. Subsequent to 3 months of oral anticoagulation therapy, the hypervascular mass in the right adnexa and the thrombosis in the right ovarian vein disappeared, which implied a complete response to treatment.
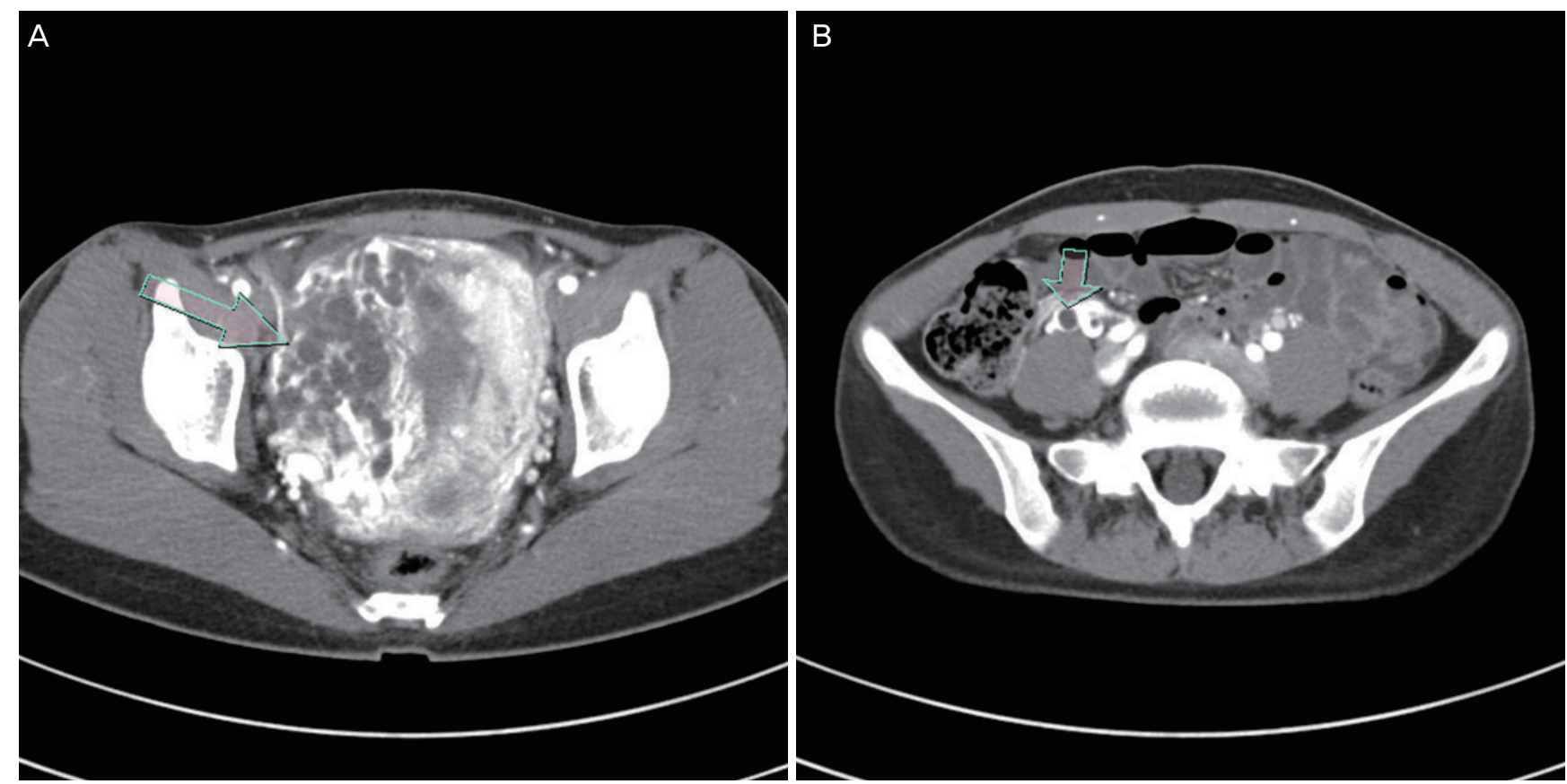

Fig. 2. Abdominal and pelvic contrast-enhanced computed tomography findings. (A) The multilobular and tortuous tubular hypodense mass with strong enhancing septae and peripheral wall in the right adnexa (arrow). (B) The right ovarian vein thrombus (arrow). 


\title{
Obstetrics \& Gynecology Science
}

\author{
Vol. 62, No. 3, 2019
}

\section{Discussion}

In 1956, Austin [9] reported the first case of OVT in a postpartum patient. OVT is a rare disease, and most often associated with the postpartum period. OVT is also associated with malignancies, pelvic inflammatory disease, inflammatory bowel disease, sepsis, and recent pelvic surgeries. However, no cases of OVT in GTN patients have been reported.

The pathophysiology of OVT can be explained by the triad of Virchow: vascular wall damage, venous stasis, and hypercoagulation. Pregnancy and puerperium are known to increase the risk of intrauterine thrombosis. The commonest cause of OVT in our patients is compression of the inferior vena cava (IVC) by the increasing size of the uterus and the possibility of blood clots forming in the deep veins of the lower limb and pelvis due to hormonal changes [10].

Symptoms typically include abdominal pain and fever. In some cases, there may be a palpable mass [11]. However, OVT presenting with only acute abdominal pain is unlikely to be suspected in GTN patients, and thus is found incidentally on CT for metastatic evaluation. Therefore, imaging studies are essential to establish the diagnosis of OVT.

Three imaging modalities are available for the diagnosis of OVT: US, CT, and magnetic resonance imaging (MRI). Doppler US is cost-effective, noninvasive, and readily available. However, the diagnostic information obtained is limited by the overlying intestinal gas and adiposity, and is highly examinerdependent. Moreover, doppler US in the case of suspected OVT has the lowest sensitivity and specificity of $55.6 \%$ and $41.5 \%$, respectively. Intravenous contrast-enhanced CT has a sensitivity and specificity of $77.8 \%$ and $62.5 \%$, respectively. MRI has the highest sensitivity and specificity of nearly $100 \%$ each [12]. However, MRI is the most expensive when compared to other modalities and is time consuming; it also has the disadvantage that it cannot be used when metal objects are inside the body. Therefore, CT scan is recommended for the initial evaluation in GTN patients who have symptoms such as abdominal pain and fever; it is also a useful method for follow-up of the disease.

Complications are rare, but they can be fatal. Thrombi can expand to the IVC, and pulmonary embolism (PE) can eventually lead to death. The incidence of PE in untreated OVT patients is about $25 \%$ and the mortality rate is about $4 \%$ [11].

The use of anticoagulants is the main treatment. Wysokin- ska et al. [13] proposed the application of the current treatment guidelines for lower extremity deep vein thrombosis (DVT) to these patients based on similar clinical outcomes for recurrent venous thromboembolism (VTE) and overall survival after anticoagulant therapy in OVT and DVT patients.

The treatment guidelines for patients with acute isolated distal DVT of the leg presenting with severe symptoms or risk factors for extension suggest initial anticoagulation therapy over serial imaging of the deep veins [14]. Traditionally, heparin and warfarin have been used. However, a few case reports suggest that the novel oral anticoagulants (NOACs) offer advantages over traditional VTE therapies. It is known that NOACs cause less massive bleeding events and do not require coagulation monitoring. Furthermore, the EINSTEIN study demonstrates that the use of rivaroxaban, a NOAC directly inhibiting factor $\mathrm{Xa}$, was linked with less bleeding events, thus improving the benefit-to-risk profile of anticoagulation therapy [15].

There is no clear guideline for the duration of anticoagulant use in OVT patients. However, the treatment guidelines for patients with an isolated distal DVT of the leg provoked by surgery or by a nonsurgical transient risk factor suggest treatment with anticoagulants for 3 months rather than for a shorter period [14].

To our knowledge, our case is the first case of OVT in GTN to be reported. She received rivaroxaban, an oral anticoagulant, for 3 months along with MTX chemotherapy. Following therapy, the abdominal CT scan showed an improvement in the OVT findings and the oral anticoagulants were discontinued. She is being followed up without recurrence for 1 year post treatment.

In conclusion, OVT is a rare disease with life-threatening complications. No cases of OVT have been reported in GTN patients. Early recognition of the condition is most important and a diagnosis of OVT must be considered when there is unexplained abdominal pain, fever, and leukocytosis during GTN diagnosis and treatment. CT scans for initial evaluation are the most useful imaging tests when symptoms are present. Overlooking this condition can lead to life-threatening complications, such as PE, sepsis, and even death. Therefore, a high level of suspicion is required for the rapid diagnosis of OVT. 


\section{Obstetrics \& Gynecology Science}

In Young Kim, et al. A rare case of ovarian vein thrombosis

\section{Conflict of interest}

No potential conflict of interest relevant to this article was reported.

\section{Patient consent}

The patients provided written informed consent for the publication and the use of their images.

\section{References}

1. Brown TK, Munsick RA. Puerperal ovarian vein thrombophlebitis: a syndrome. Am J Obstet Gynecol 1971;109:263-73.

2. André M, Delèvaux I, Amoura Z, Corbi P, Courthaliac C, Aumaître $\mathrm{O}$, et al. Ovarian vein thrombosis in the antiphospholipid syndrome. Arthritis Rheum 2004;50:1836.

3. Heavrin BS, Wrenn K. Ovarian vein thrombosis: a rare cause of abdominal pain outside the peripartum period. J Emerg Med 2008;34:67-9.

4. Jacoby WT, Cohan RH, Baker ME, Leder RA, Nadel SN, Dunnick NR. Ovarian vein thrombosis in oncology patients: CT detection and clinical significance. AJR Am J Roentgenol 1990;155:291-4.

5. Klima DA, Snyder TE. Postpartum ovarian vein thrombosis. Obstet Gynecol 2008;111:431-5.

6. Marcovici I, Goldberg E. Ovarian vein thrombosis associated with Crohn's disease: a case report. Am J Obstet Gynecol 2000;182:743-4.

7. Salomon O, Apter S, Shaham D, Hiller N, Bar-Ziv J,
Itzchak $Y$, et al. Risk factors associated with postpartum ovarian vein thrombosis. Thromb Haemost 1999;82:1015-9.

8. Simons GR, Piwnica-Worms DR, Goldhaber SZ. Ovarian vein thrombosis. Am Heart J 1993;126:641-7.

9. Austin OG. Massive thrombophlebitis of the ovarian veins; a case report. Am J Obstet Gynecol 1956;72:4289.

10. Calderwood CJ, Jamieson R, Greer IA. Gestational related changes in the deep venous system of the lower limb on light reflection rheography in pregnancy and the puerperium. Clin Radiol 2007;62:1174-9.

11. Harris K, Mehta S, Iskhakov E, Chalhoub M, Maniatis T, Forte $F$, et al. Ovarian vein thrombosis in the nonpregnant woman: an overlooked diagnosis. Ther Adv Hematol 2012;3:325-8.

12. Kubik-Huch RA, Hebisch $G$, Huch $R$, Hilfiker P, Debatin JF, Krestin GP. Role of duplex color Doppler ultrasound, computed tomography, and MR angiography in the diagnosis of septic puerperal ovarian vein thrombosis. Abdom Imaging 1999;24:85-91.

13. Wysokinska EM, Hodge D, McBane RD 2nd. Ovarian vein thrombosis: incidence of recurrent venous thromboembolism and survival. Thromb Haemost 2006;96:12631.

14. Kearon C, Akl EA, Ornelas J, Blaivas A, Jimenez D, Bounameaux $\mathrm{H}$, et al. Antithrombotic therapy for VTE disease: CHEST guideline and expert panel report. Chest 2016;149:315-52.

15. EINSTEIN Investigators, Bauersachs R, Berkowitz SD, Brenner B, Buller HR, Decousus $H$, et al. Oral rivaroxaban for symptomatic venous thromboembolism. N Engl J Med 2010;363:2499-510. 